\title{
EXPONENTIAL WINDOW FAMILY
}

\author{
Kemal Avci ${ }^{1}$ and Arif Nacaroglu ${ }^{2}$ \\ ${ }^{1}$ Department of Electrical and Electronics Engineering, \\ Abant Izzet Baysal University, Bolu, Turkey \\ avci_keibu.edu.tr \\ ${ }^{2}$ Department of Electrical and Electronics Engineering, \\ University of Gaziantep, Gaziantep, Turkey \\ arif1@gantep.edu.tr
}

\begin{abstract}
In this paper we propose a new class of 2-parameter adjustable windows, namely Exponential window, based on the exponential function [1,2]. The Exponential window is derived in the same way as Kaiser window was derived, but our proposed window is more computationally efficient because in its time domain function it has no power series expansion. First, the spectrum design equations for the Exponential window are established, and the spectral comparisons are performed with Cosh, Kaiser and ultraspherical windows. The proposed window is compared with Cosh and Kaiser windows, and the results show that for the same window length and mainlobe width the Exponential window provides better sidelobe roll-off ratio characteristic, which may be important for some applications, but worse ripple ratio. The second comparison is performed with ultraspherical window for the same window length, mainlobe width and sidelobe roll-off ratio and the results demonstrate that the Exponential window exhibits better ripple ratio for the narrower mainlobe width and larger sidelobe roll-off ratio, but worse ripple ratio for the wider mainlobe width and smaller sidelobe roll-off ratio.
\end{abstract}

\section{KEYWORDS}

Window function, Exponential window, Cosh Window, Kaiser window, Ultraspherical window

\section{INTRODUCTION}

Providing new window functions (or simply as windows) is in interest, because they are widely used in digital signal processing applications, e.g., signal analysis and estimation, digital filter design and speech processing [1-3]. In literature many windows have been proposed [4-16]. Since the best window depends on the applications, they are known as suboptimal solutions.

Kaiser window [5] is a well-known two parameter flexible window and widely used for FIR filter design and spectrum analysis applications. It performs good results because it achieves close approximation to the discrete prolate spheroidal functions that have maximum energy concentration in the mainlobe. With adjusting its two independent parameters, the window length and the shape parameter, it can control the spectral parameters main lobe width and ripple ratio for various applications.

Sidelobe roll-off ratio, which is important for some applications, is another window spectral parameter to differentiate the performances of the windows. For beamforming applications, the higher sidelobe roll-off ratio means that it can reject far end interferences better [11]. For the design of nonrecursive digital filters, it reduces the far end attenuation for stopband energy [12], and reducing the energy leak from one band to another for speech processing [17].

In terms of roll-off ratio characteristic the Kaiser window provides better sidelobe than the other well-known two parameter adjustable windows such as Dolph-Chebyshev [4] and Saramaki [6].

DOI : 10.5121/sipij.2013.4401 
Signal \& Image Processing : An International Journal (SIPIJ) Vol.4, No.4, August 2013

Therefore, providing a window performing higher sidelobe roll-off characteristic than the Kaiser window will be useful for some signal processing applications.

In this paper, a new window based on the exponential function is proposed to provide higher sidelobe roll-off ratio than Kaiser window to be useful for some applications.

\section{DERIVATION OF THE EXPONENTIAL WINDOW}

In this section, a brief explanation about how to derive the proposed window function is given.

\subsection{Windows}

An N-length window, denoted by $w(n T)$, is a time domain function which is nonzero for $n \leq \mid$ (N1)/2 $\mid$ and zero for otherwise. They are generally compared and classified in terms of their spectral characteristics. The frequency spectrum of $w(n T)$ can be found by

$$
W\left(e^{j w T}\right)=|A(w)| e^{j \theta(w)}=w(0)+2 \sum_{n=1}^{(N-1) / 2} w(n T) \cos w n T
$$

where $\mathrm{T}$ is the sample period. A typical window has a normalized amplitude spectrum in $\mathrm{dB}$ range as in Figure 1.

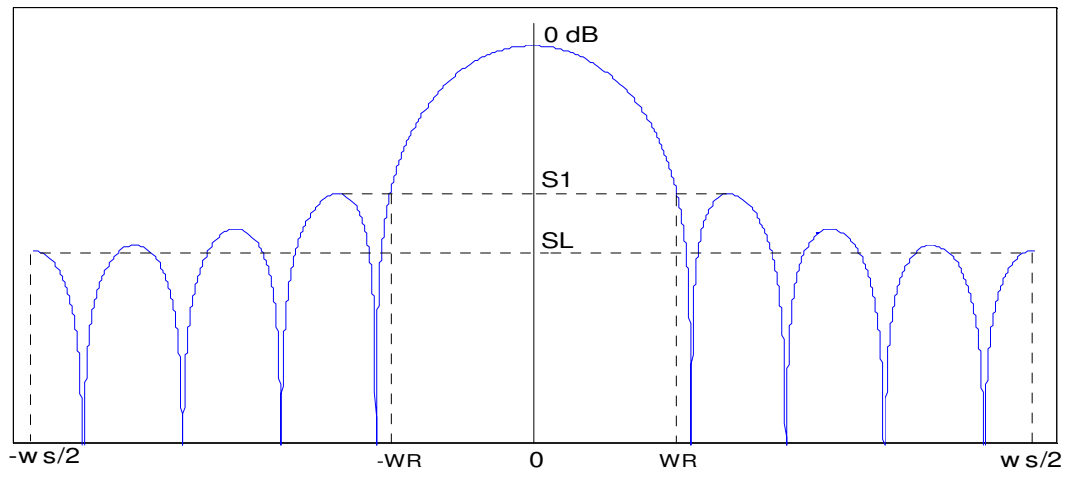

Figure 1. A typical window's normalized amplitude spectrum

Normalized spectrum in Fig.1 can be obtained from

$$
\left|W_{N}\left(e^{j w T}\right)\right|=20 \log _{10}\left(|A(w)| /|A(w)|_{\max }\right)
$$

The common spectral characteristic parameters to distinguish the windows performance are the mainlobe width $\left(\mathrm{w}_{\mathrm{M}}\right)$, the ripple ratio $(\mathrm{R})$ and the sidelobe roll-off ratio $(\mathrm{S})$. From Figure 1, these parameters can be defined as

$\mathrm{w}_{\mathrm{M}}=$ Two times half mainlobe width $=2 \mathrm{w}_{\mathrm{R}}$

$\mathrm{R}=$ Maximum sidelobe amplitude in $\mathrm{dB}$ - Mainlobe amplitude in $\mathrm{dB}=\mathrm{S}_{1}$

$\mathrm{S}=$ Maximum sidelobe amplitude in $\mathrm{dB}$ - Minimum sidelobe amplitude in $\mathrm{dB}=\mathrm{S}_{1}-\mathrm{S}_{\mathrm{L}}$

In the applications, it is desired for a window to have a smaller ripple ratio and a narrower mainlobe width. But, this requirement is contradictory [3].

\subsection{Kaiser Window}

Kaiser window is defined in discrete time, as [3,5] 
Signal \& Image Processing : An International Journal (SIPIJ) Vol.4, No.4, August 2013

$$
w_{k}(n)=\left\{\begin{array}{cc}
\frac{I_{0}\left(\alpha_{k} \sqrt{1-\left(\frac{2 n}{N-1}\right)^{2}}\right)}{I_{0}\left(\alpha_{k}\right)} & |n| \leq \frac{N-1}{2} \\
0 & \text { otherwise }
\end{array}\right.
$$

where $\alpha_{\mathrm{k}}$ is the adjustable shape parameter, and $\mathrm{I}_{0}(\mathrm{x})$ is the modified Bessel function of the first kind of order zero, which is described by the following power series expansion as

$$
I_{0}(x)=1+\sum_{k=1}^{\infty}\left[\frac{1}{k !}\left(\frac{x}{2}\right)^{k}\right]^{2}
$$

While an approximation closed formula for the Kaiser window spectrum is defined [3], the exact Kaiser spectrum can be obtained from Eq. (1). Note that $\mathrm{T}=1$ is considered as the normalization for the rest of paper.

As known from the fixed windows while the window length, $\mathrm{N}$, increases the mainlobe width decreases but ripple ratio remains generally constant. And, larger values of the shape parameter, $\alpha_{\mathrm{k}}$, result in a wider mainlobe width and a smaller ripple ratio.

\subsection{Exponential Window}

From Figure 2, it can be seen that $\exp (\mathrm{x})$ and $\operatorname{Io}(\mathrm{x})$ have the same shape characteristic.

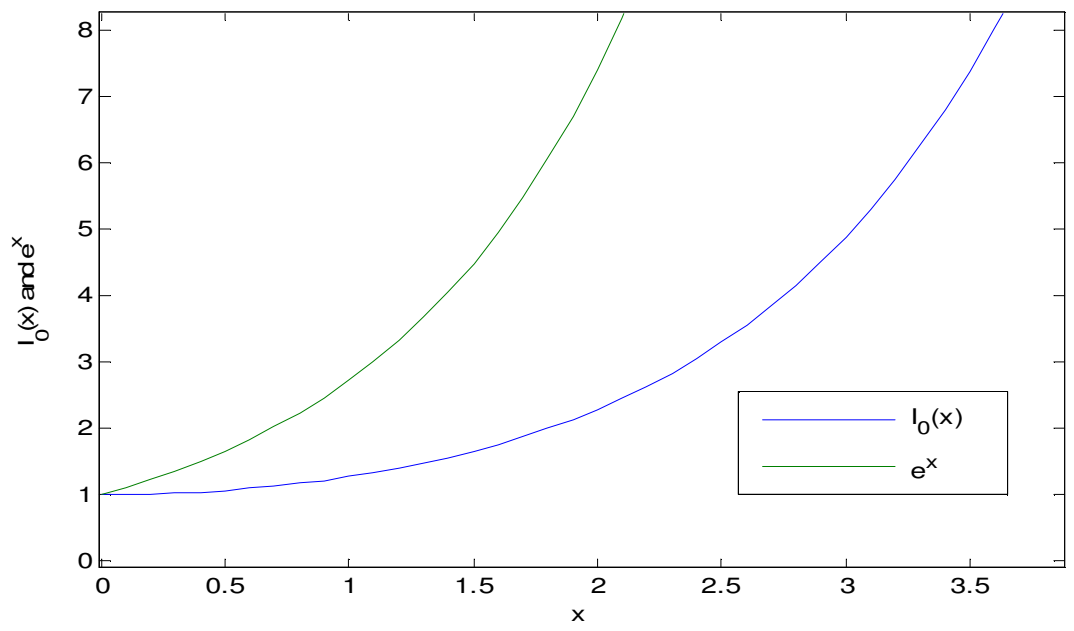

Figure 2. The functions $\operatorname{Io}(\mathrm{x})$ and $\mathrm{e}^{\mathrm{x}}$

Therefore, a new window, called "Exponential window” for this paper, can be proposed as

$$
w_{e}(n)=\left\{\begin{array}{cc}
\frac{e^{\left(\alpha_{e} \sqrt{1-\left(\frac{2 n}{N-1}\right)^{2}}\right)}}{e^{\alpha_{e}}} & |n| \leq \frac{N-1}{2} \\
0 & \text { otherwise }
\end{array}\right.
$$


Signal \& Image Processing : An International Journal (SIPIJ) Vol.4, No.4, August 2013

Like the Kaiser window, the Exponential window has two independent parameters, namely the window length $(\mathrm{N})$ and the adjustable shape parameter $\left(\alpha_{\mathrm{e}}\right)$. Figure 3 shows the time domain characteristic of the exponential window for various values of the parameter $\alpha_{\mathrm{e}}$ with $\mathrm{N}=51$. It is seen that $\alpha_{\mathrm{e}}=0$ corresponds to the rectangular window as in the case for the Kaiser window. For larger values of $\alpha_{e}$, the Exponential window becomes to have a Gaussian shape.

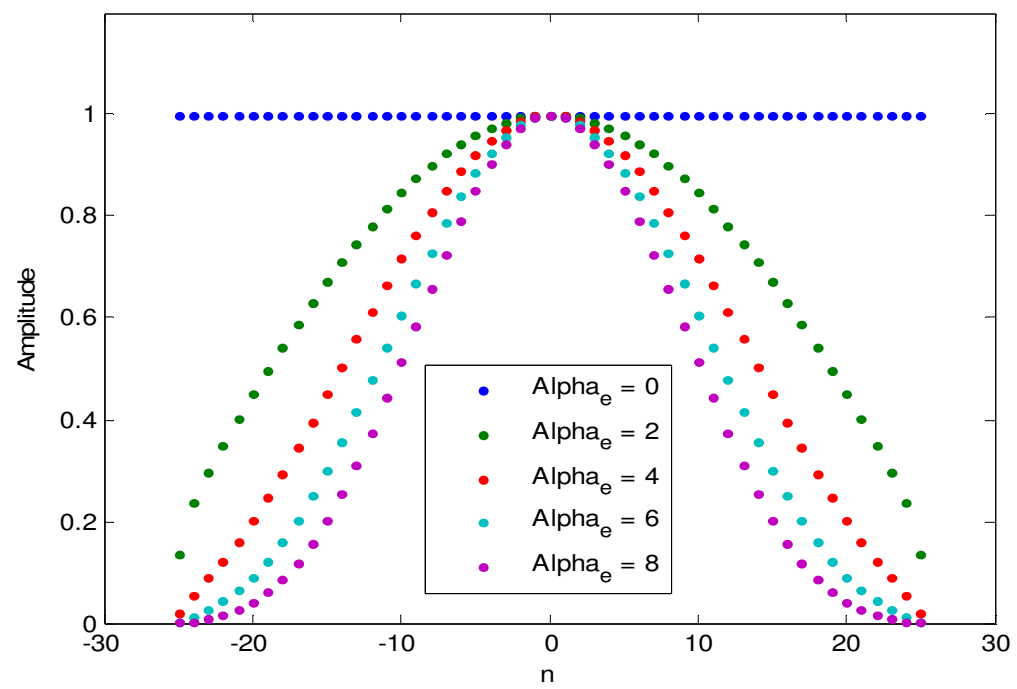

Figure 3. Exponential window in time domain for $\alpha_{\mathrm{e}}=0,2,4,6$, and 8 with $\mathrm{N}=51$

The exact spectrum for the Exponential window can be obtained from Eq. (1). Figure 4 shows the effect of $\alpha_{e}$ on the Exponential window spectrum for a fixed value of length $N=51$. And, Table 1 summarizes the numerical data in Figure 4. As seen from the figure and table, an increase in $\alpha_{e}$ results in a wider mainlobe width and a smaller ripple ratio.

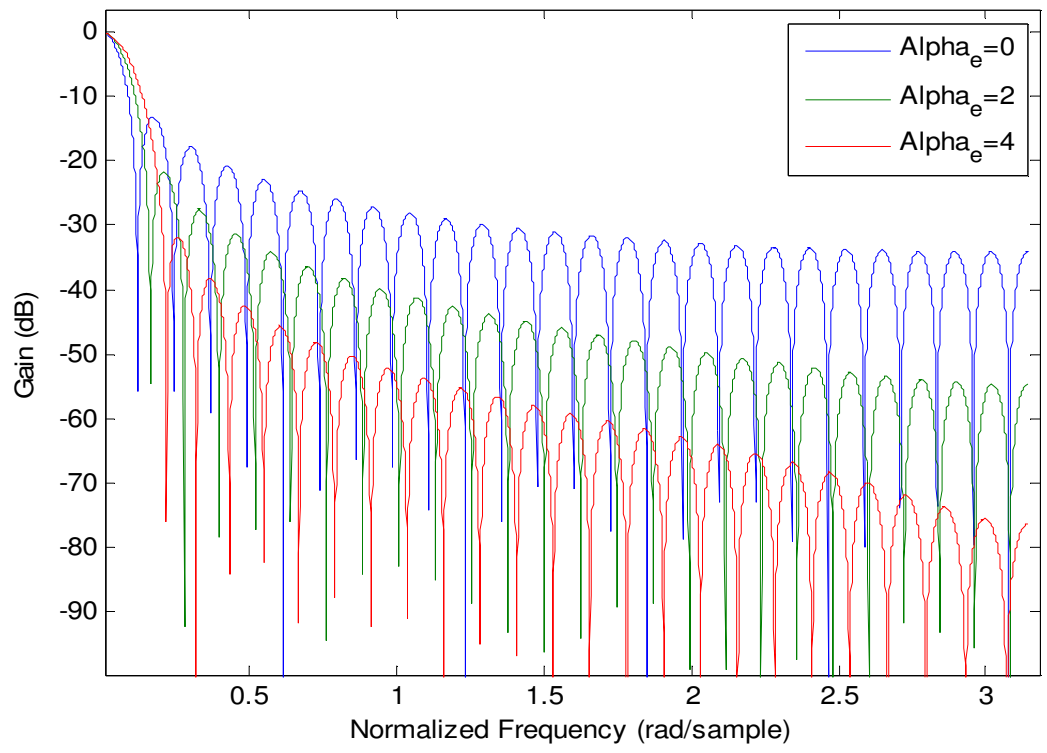

Figure 3. Proposed window spectrum in $\mathrm{dB}$ for $\alpha=0,2$, and 4 and $\mathrm{N}=51$ 
Signal \& Image Processing : An International Journal (SIPIJ) Vol.4, No.4, August 2013

Table 1. Spectral data for Exponential window

\begin{tabular}{|l|l|l|l|l|l|}
\hline Window & $\mathrm{N}$ & $\alpha$ & $\mathrm{w}_{\mathrm{R}}$ & $\mathrm{R}$ & $\mathrm{S}$ \\
\hline Proposed-1 & 51 & 0 & 0.1 & -13.25 & 20.9 \\
\hline Proposed-2 & 51 & 2 & 0.15 & -21.73 & 32.95 \\
\hline Proposed-3 & 51 & 4 & 0.21 & -31.84 & 44.54 \\
\hline
\end{tabular}

\section{SPECTRUM DESIGN EQUATIONS}

It is important for some applications such as the spectrum analysis to have the window design equations which define the window parameters in terms of the spectral parameters.

To obtain the spectrum design equations for the Exponential window, it is necessary to find the relations between the window parameters and spectral parameters empirically. Figure 4 shows the relation between $\alpha_{\mathrm{e}}$ and the ripple ratio for the window lengths $\mathrm{N}=51$ and 101 .

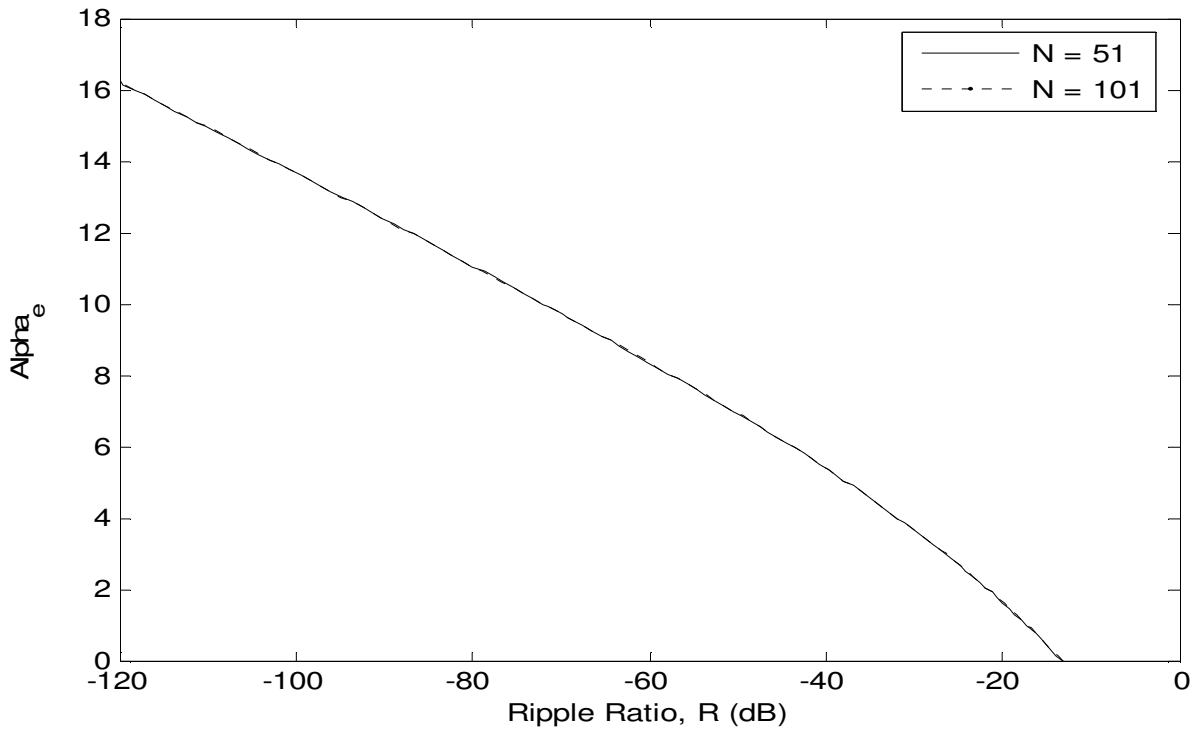

Figure 4. Relation between $\alpha_{\mathrm{e}}$ and $\mathrm{R}$ for the Exponential window with $\mathrm{N}=51$ and 101

It is seen from Figure 4 that the window length parameter doesn't affect the relation between the adjustable parameter $\alpha_{e}$ and the ripple ratio. Therefore, using the curve fitting method in MATLAB, the first design equation for $\alpha_{\mathrm{e}}$ in terms of the ripple ratio can be obtained as

$$
\alpha_{e, A p p r}=\left\{\begin{array}{cc}
0 & R>-13.26 \\
-1.513 \times 10^{-3} R^{2}-0.2809 R-3.398 & -50<R \leq-13.26 \\
-1.085 \times 10^{-4} R^{2}-0.1506 R-0.304 & -120 \leq R \leq-50
\end{array}\right.
$$

The quadratic approximation model given by Eq. (6) for the adjustable parameter $\alpha_{\mathrm{e}}$ is plotted in Figure 5. It is seen that the proposed model provides a good approximation for $\mathrm{N}=101$. Moreover, the approximation error for the first design equation for $\mathrm{N}=101$ is plotted in Figure 6. It is observed that the amplitude of deviations in the alpha is lower than 0.06 which corresponds to very small error in the ripple ratio. 
Signal \& Image Processing : An International Journal (SIPIJ) Vol.4, No.4, August 2013

More accurate results can be obtained by restricting the range or using higher order approximations, but the proposed model for the Exponential window is adequate for most applications like the Kaiser model.

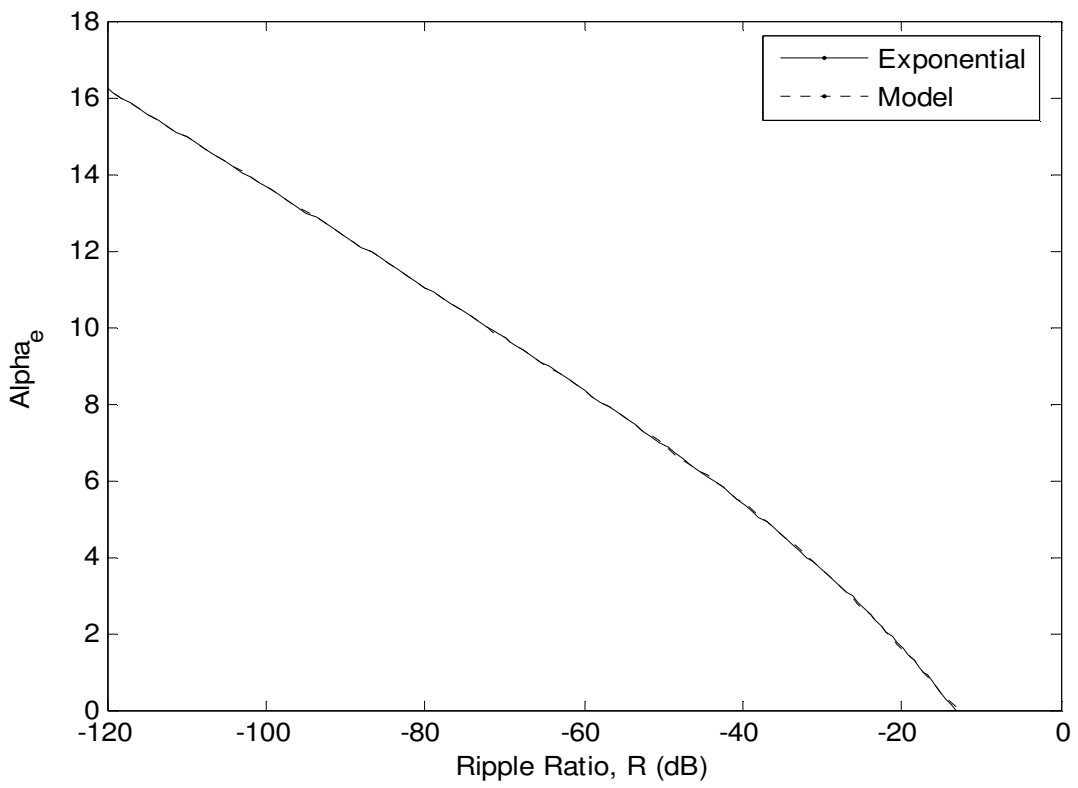

Figure 5. Approximated model for $\alpha_{\mathrm{e}}$ of the Exponential window with $\mathrm{N}=101$

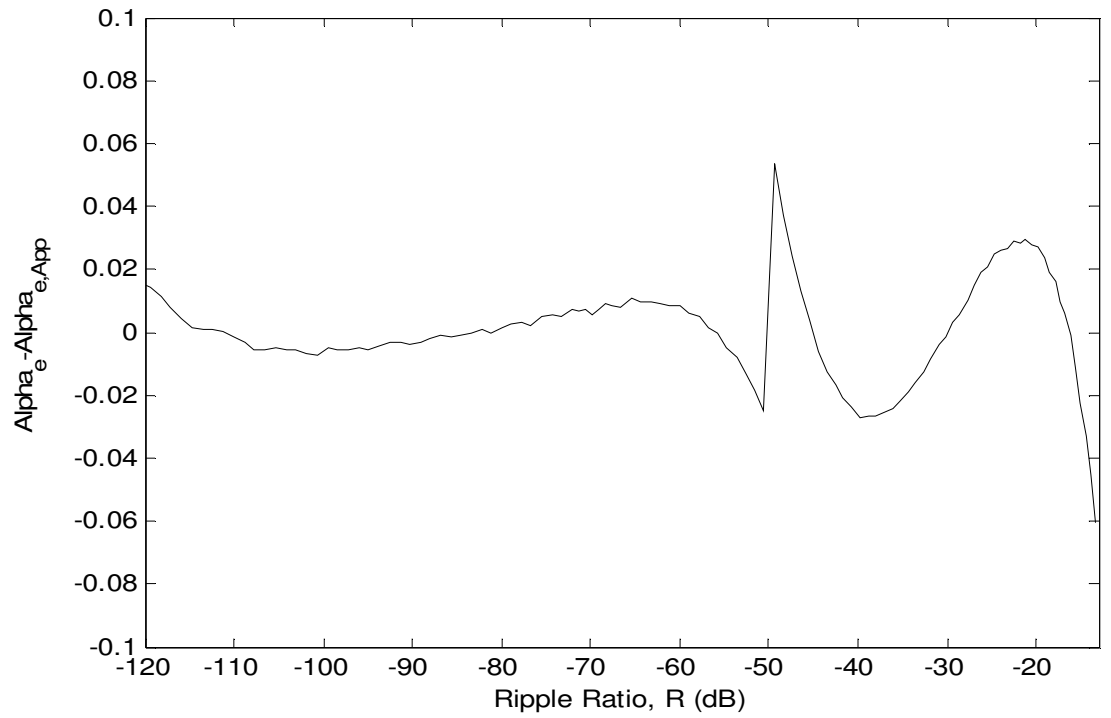

Figure 6. Error curve of approximated $\alpha_{e}$ versus $R$ for $\mathrm{N}=101$

The second design equation is the relation between the window length and the ripple ratio. To predict the window length for a given quantities $R$ and $w_{R}$, the normalized width parameter $D_{w}=$ $2 \mathrm{w}_{\mathrm{R}}(\mathrm{N}-1)$ is used [11]. The relation between $\mathrm{D}_{\mathrm{w}}$ and $\mathrm{R}$ for the Exponential window with $\mathrm{N}=51$ and 101 is plotted in Figure 7. 
Signal \& Image Processing : An International Journal (SIPIJ) Vol.4, No.4, August 2013

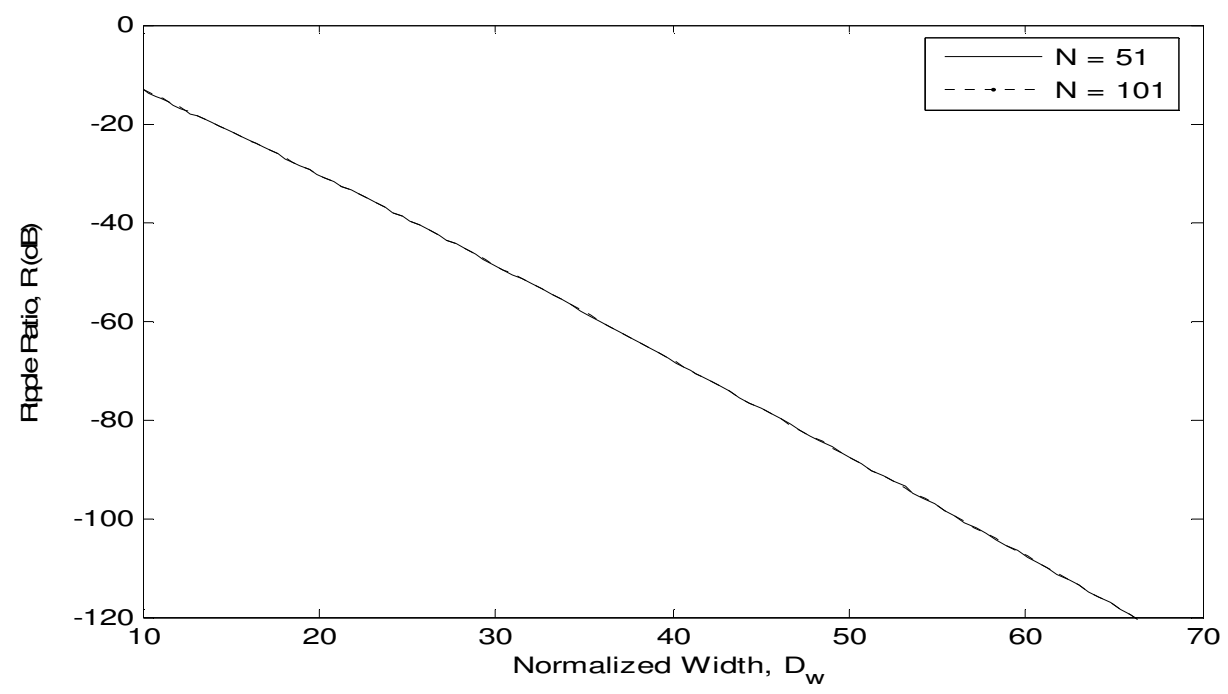

Figure 7. Relation between $D_{w}$ and $R$ for the Exponential window with $\mathrm{N}=51$ and 101

It is seen from Figure 7 that as the ripple ratio becomes smaller the mainlobe width becomes wider. Also, it is observed from the same figure that the window length has no effect on the relation between the ripple ratio and normalized mainlobe width. By using the curve fitting method, an approximate design relationship between the normalized width $\left(D_{w}\right)$ and the ripple ratio $(R)$ can be established as

$$
D_{w, \text { Appr }}=\left\{\begin{array}{cc}
0 & R>-13.26 \\
-7.58 \times 10^{-5} R^{3}+7.22 \times 10^{-3} R^{2}-0.3566 R+4.312 & -50<R \leq-13.26 \\
-1.297 \times 10^{-4} R^{2}-0.5281 R+4.708 & -120 \leq R \leq-50
\end{array}\right.
$$

The approximation model given by Eq. (7) for the normalized mainlobe width is plotted in Figure 8. It is seen that the proposed model provides a good approximation for $\mathrm{N}=101$.

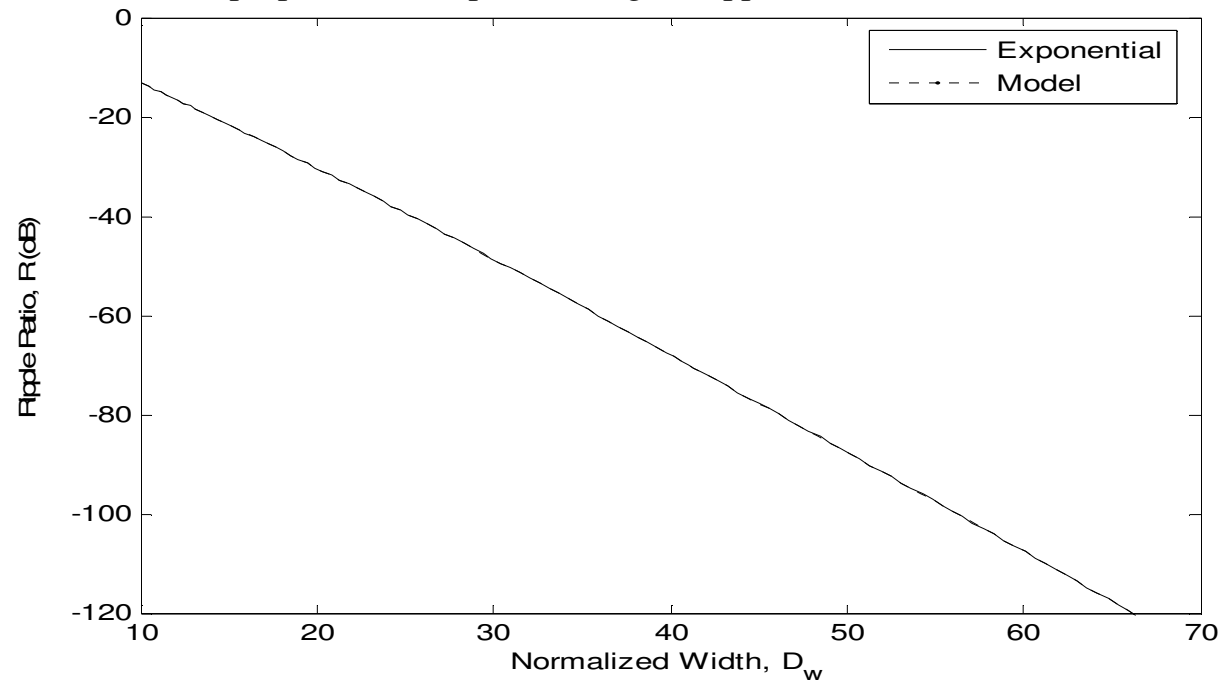

Figure 8. Approximated model for $\mathrm{D}_{\mathrm{w}}$ of the Exponential window with $\mathrm{N}=101$ 
Signal \& Image Processing : An International Journal (SIPIJ) Vol.4, No.4, August 2013

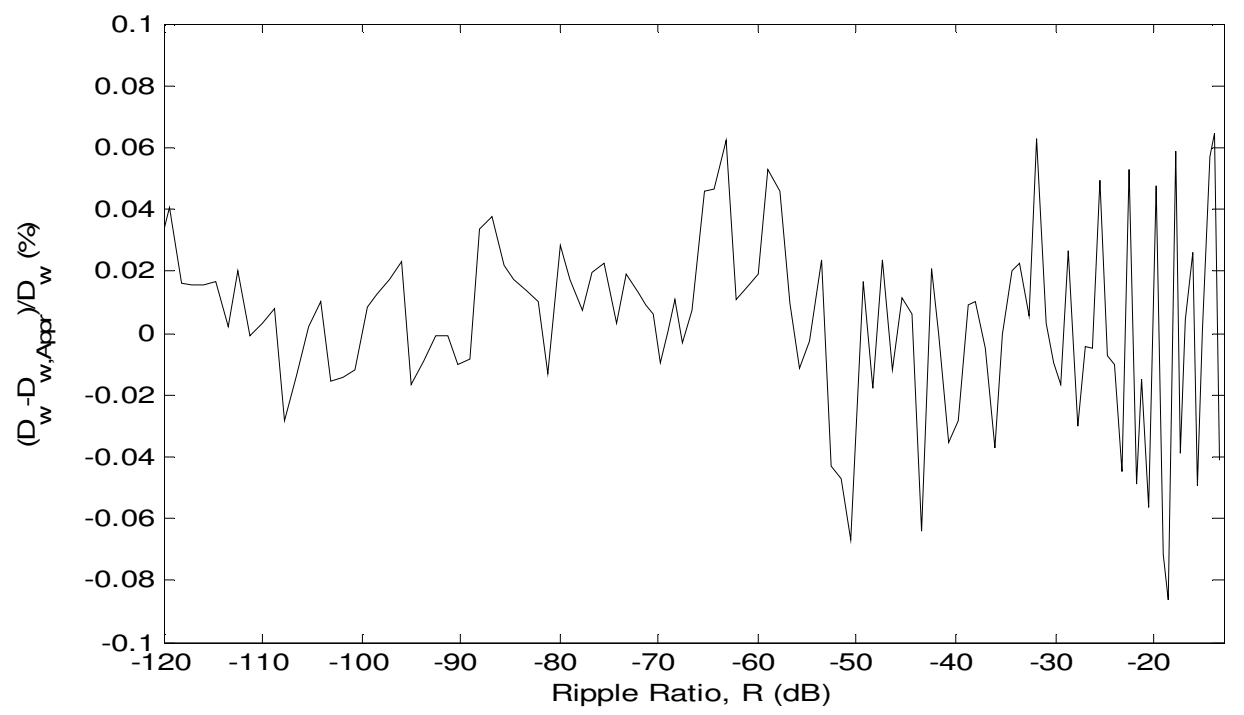

Figure 9. Relative error of approximated $D_{w}$ for the Exponential window in percent versus $R$ with $\mathrm{N}=101$

The relative error of approximated normalized width in percent versus the ripple ratio for $\mathrm{N}=101$ is plotted in Figure 9. The percentage error in the model changes between 0.065 and -0.086 . This error range satisfies the error criterion in [11] which states that the predicted error in the normalized width must be smaller than $1 \%$.

An integer value of the window length $N$ can be predicted from [11]

$$
N \geq \frac{D_{w, A p p r}}{2 w_{R}}+1
$$

Using the equations (6) through (8), an Exponential window can be designed for satisfying the given prescribed values of the ripple ratio and mainlobe width.

In some applications [17], larger sidelobe roll-off ratio may be desired. Figure 10 shows the change in the sidelobe-roll off ratio in terms of the normalized mainlobe width parameter for $\mathrm{N}=$ 51 and 101. From the figure it can be seen that the sidelobe roll off ratio becomes larger as normalized width increases until one of the sidelobes is dropped due to higher value of alpha. Unlike in the case of ripple ratio, a change in the window length affects significantly the sidelobe roll-off ratio characteristic of the Exponential window.

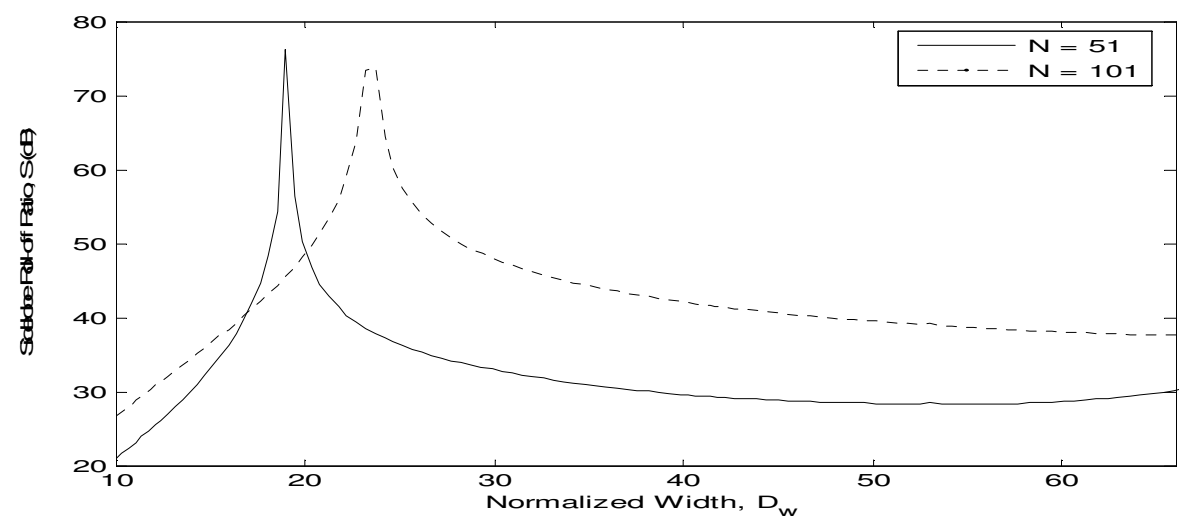

Figure 10. Relation between $D_{w}$ and $\mathrm{S}$ for the Exponential window with $\mathrm{N}=51$ and 101 
Signal \& Image Processing : An International Journal (SIPIJ) Vol.4, No.4, August 2013

\section{SPECTRUM COMPARISON EXAMPLES}

\subsection{Comparison with Kaiser and Cosh Windows}

Figure 11 shows a general comparison of the Cosh window in a wide range with the Exponential and Kaiser windows in terms of the ripple ratio versus normalized mainlobe width for $\mathrm{N}=101$. The figure demonstrates that the Kaiser window provides smaller ripple ratio than the others for the same mainlobe width. For the range $D_{w}<25$, the Cosh window produces smaller ripple ratio than the Exponential window. And, for the range $25<\mathrm{D}_{\mathrm{w}}$ the Cosh and Exponential windows perform the same ripple ratio characteristic.

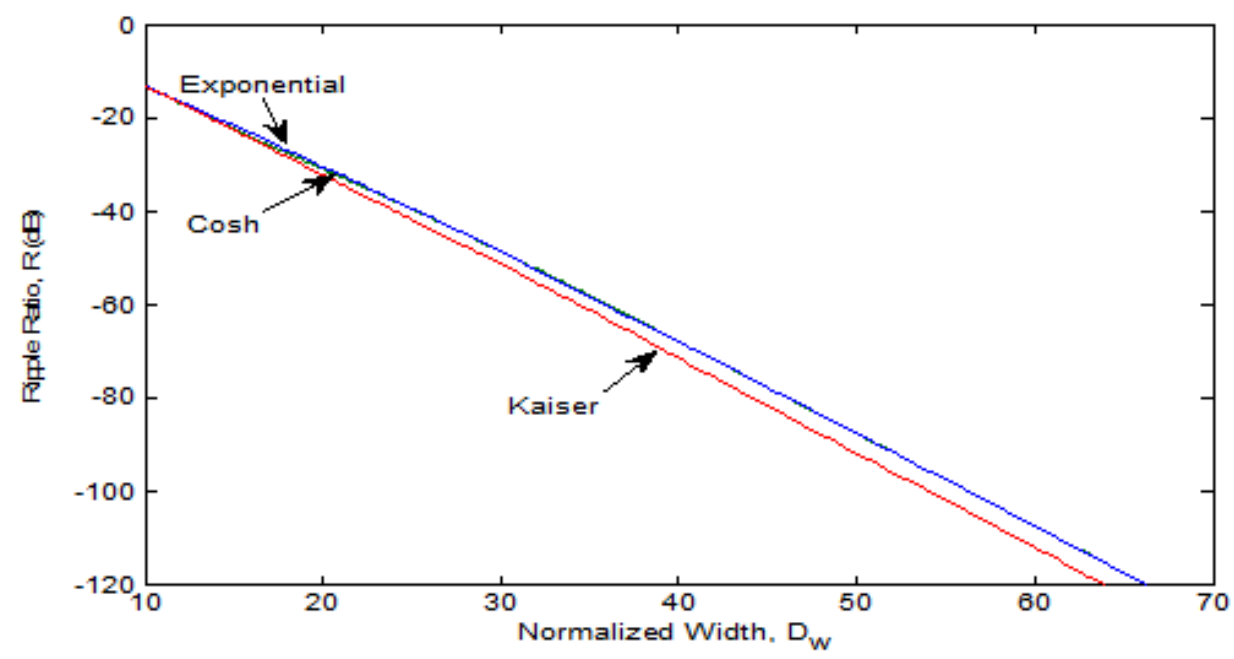

Figure 11. Ripple ratio comparison between the Cosh, Exponential and Kaiser windows for $\mathrm{N}=101$

The simulation results for the sidelobe roll-off ratio comparison is given for $\mathrm{N}=101$ in Figure 12 . It is seen that the Cosh window performs better than the Kaiser window but worse than the Exponential window in terms of the sidelobe roll-off ratio for the same mainlobe width until one sidelobe is lost where the peak values occur.

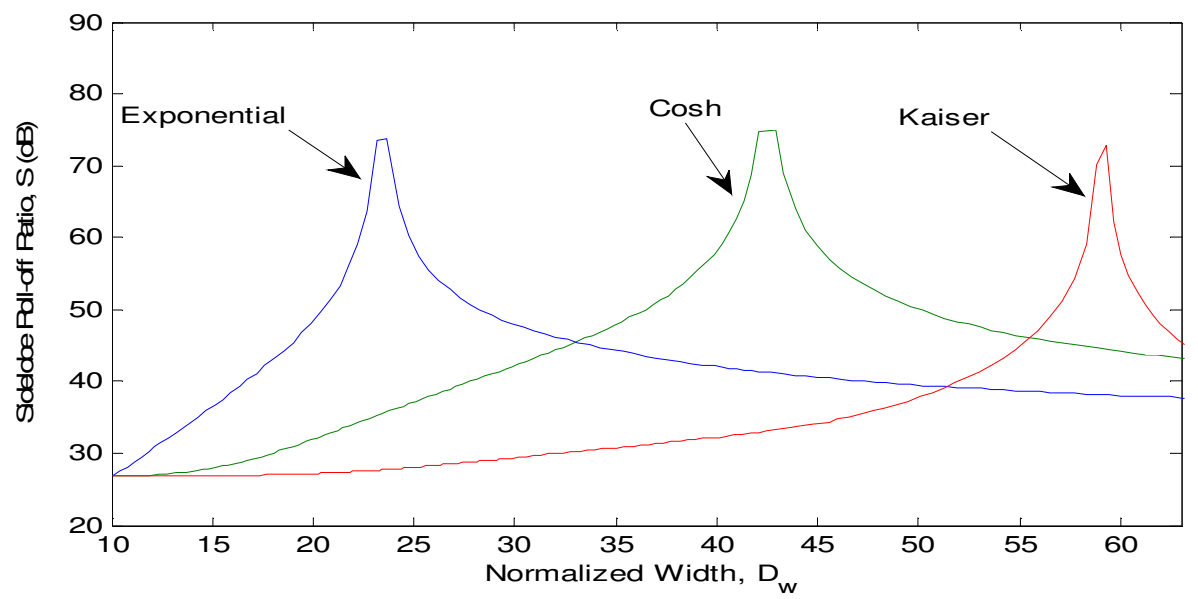

Figure 12. Sidelobe roll-off ratio comparison between the Cosh, Exponential and Kaiser windows for $\mathrm{N}=101$ 


\subsection{Comparison with Ultraspherical Window}

Two specific examples are given for the comparison between the Exponential and ultraspherical windows. The first comparison example is performed for the narrower mainlobe width and larger sidelobe roll-off ratio with $\mathrm{N}=51$.

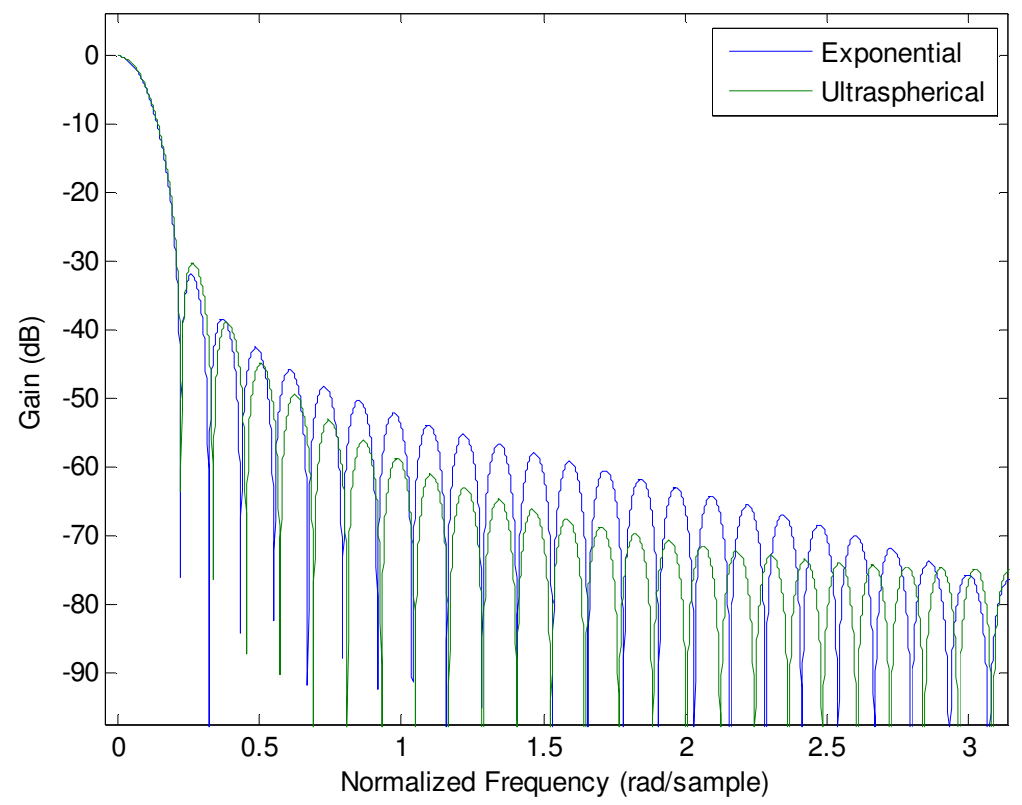

Figure 13. Comparison of the proposed and ultraspherical windows for narrower mainlobe width and larger sidelobe roll-off ratio for $\mathrm{N}=51$

The simulation result given in Figure 13 and Table 2 which summarizes the figure shows that the three-parameter ultraspherical window provides a better ripple ratio than the Exponential window for the same window length, mainlobe width and sidelobe roll-off ratio. The ultraspherical window parameters for this example are $\mu=1.99999$ and $x_{\mu}=1.00039$.

Table 2. Data for the first comparison example

\begin{tabular}{|l|l|l|l|l|}
\hline Window & $\mathrm{N}$ & $\mathrm{w}_{\mathrm{R}}$ & $\mathrm{S}$ & $\mathrm{R}$ \\
\hline Exponential & 51 & 0.164 & 37.81 & -24.1 \\
\hline Ultraspherical & 51 & 0.164 & 37.81 & -23.02 \\
\hline
\end{tabular}

The second comparison example is given for the wider mainlobe width and smaller sidelobe rolloff ratio for $\mathrm{N}=51$. The simulation result given in Figure 14 and Table 3 shows that the Exponential window provides a better ripple ratio than the ultraspherical window in this case. The ultraspherical window parameters for this example are $\mu=1.66635$ and $x_{\mu}=1.00973$. 


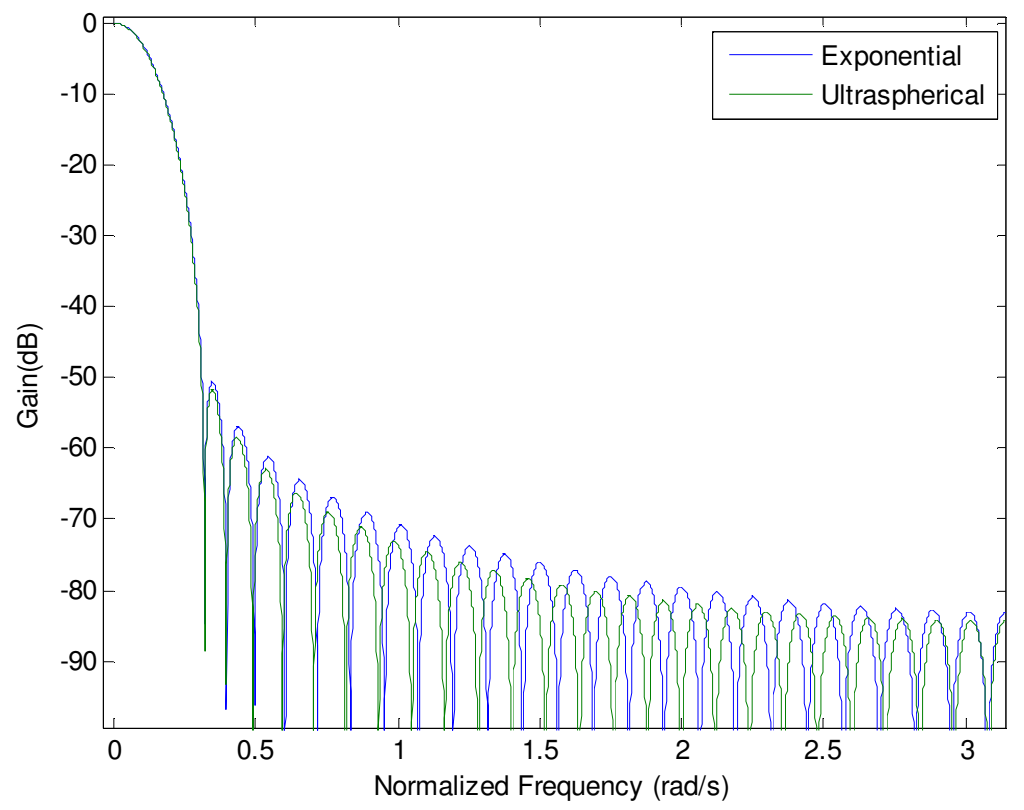

Figure 14. Comparison of the proposed and ultraspherical windows for wider mainlobe width and smaller sidelobe roll-off ratio for $\mathrm{N}=51$

From Figures 13 and 14, the ripples between the maximum and the minimum sidelobe amplitudes can also be observed to be higher for the Exponential window.

Table 3. Data for the first comparison example

\begin{tabular}{|l|l|l|l|l|}
\hline Window & $\mathrm{N}$ & $\mathrm{w}_{\mathrm{R}}$ & $\mathrm{S}$ & $\mathrm{R}$ \\
\hline Proposed & 51 & 0.31 & 32.48 & -50.53 \\
\hline Ultraspherical & 51 & 0.31 & 32.48 & -51.75 \\
\hline
\end{tabular}

\section{Conclusions}

In this paper, a new 2-parameter window family based on the exponential function has been proposed. Since it's derived using the exponential function, it has been called "Exponential window" for this paper. First, the proposed window family has been introduced by giving its derivation and mathematical definition. And then, its spectrum design equations using curve fitting method in MATLAB have been obtained.

To demonstrate the performance of the proposed window, its spectral comparisons have been performed with Cosh, Kaiser and ultraspherical windows. Comparison with Cosh and Kaiser windows showed that the Exponential window provides better sidelobe roll-off ratio characteristic, but presents worse ripple ratio for the same window length and mainlobe width. As for the comparison with 3-paramater ultraspherical window, for the same the window length, mainlobe width and sidelobe roll-off ratio parameters, the Exponential window presents better ripple ratio for the narrower mainlobe width and larger sidelobe roll-off ratio, but exhibits worse ripple ratio for the wider mainlobe width and smaller roll-off ratio. 
Signal \& Image Processing : An International Journal (SIPIJ) Vol.4, No.4, August 2013

\section{REFERENCES}

[1] Avci, Kemal (2008) Design of high-quality low order nonrecursive digital filters using the window functions, $\mathrm{PhD}$ Thesis, Gaziantep University.

[2] Avci, Kemal \& Nacaroglu, Arif (2008) "A new window based on exponential function" Proc. Of IEEE Ph.D. Research in Microelectronics and Electronics (PRIME 2008). June, Istanbul, Turkey. pp 69-72.

[3] Antoniou, Andreas (2005) Digital signal processing: Signal, systems, and filters. New York: McGraw Hill.

[4] Dolph, C. L. (1946) “A Current distribution for broadside arrays which optimizes the relationship between beamwidth and side-lobe level" Proc. IRE, vol.34, June, pp 335-348.

[5] Kaiser, J.F. \& Schafer, R.W (1980) "On the use of the Io-sinh window for spectrum analysis" IEEE Trans. Acoustics, Speech, and Signal Processing, Vol. 28, No.1, pp 105-107.

[6] Saramaki, Tapio (1989) "A class of window functions with nearly minimum sidelobe energy for designing FIR filters" in Proc. IEEE Int. Symp. Circuits and systems (ISCAS'89), Portland, Ore, USA, vol.1, pp 359-362

[7] Ha, Y.H. and Pearce, J.A. (1989). "A new window and comparison to standard windows". IEEE Transactions on Acoustics, Speech, and Signal Processing. 37/2, pp 298-301.

[8] Adams, J.W. (1991) “A new optimal window”. IEEE Transactions on Signal Processing. 39/8, pp 1753-1769.

[9] Yang, S. and Ke, Y. (1992). "On the three-coefficient window family”. IEEE Transactions on Signal Processing. 40/12, 3085-3088.

[10] Gautam, J.K., \& Kumar, A. \& and Saxena, R. (1996). "On the modified Bartlett-Hanning window (family)". IEEE Transactions on Signal Processing. 44/8, pp 2098-2102.

[11] Bergen, S.W.A. \& Antoniou, Andreas (2004) "Design of ultraspherical window functions with prescribed spectral characteristics” EURASIP Journal on Applied Signal Processing, 13, pp 20532065.

[12] Sharma, S.N. \& Saxena, R. \& Saxena, S.C. (2004) "Design of FIR filter using variable window families: A comparative study” J. Indian Inst. Sci., Sept.-Oct., 84, pp 155-161.

[13] Avci, Kemal \& Nacaroglu, Arif (2009) "Cosh window family and its application to FIR filter design" AEU-Int. J. Electronics and Communications, 63, pp 907-916.

[14] Avci, Kemal \& Nacaroglu, Arif (2008) "Modification of Cosh Window Family". Proc. of Third International Conference on Information and Communication Technologies (ICTTA'08). April. Damascus, Syria, pp 291-292.

[15] Avci, Kemal \& Nacaroglu, Arif (2009) "An Efficient Study on the Modification of Kaiser Window". MTA Review. Vol. XIX, No.1

[16] Avci, Kemal (2013) "Performance Analysis of Kaiser-Hamming Window for Nonrecursive Digital Filter Design” 21. Signal Processing and Communication Applications Conference (SIUU 2013), 24-26 April, Girne, North Cyprus, pp. 1-4.

[17] Jain, A \& Saxena, R \& Saxena, S.C. (2005) “A simple alias-free QMF system with near-perfect reconstruction” J. Indian Ins. Sci., Jan-Feb, no.12, pp 1-10.

\section{Authors}

Kemal Avci was born in Adiyaman, Turkey in 1980. He received his B.S., M.S., and Ph.D. degrees in Electrical and Electronics Engineering from University of Gaziantep,Turkey in 2002, 2004, and 2008, respectively. Currently, he works as an assistant professor in Electrical and Electronics Engineering in Abant Izzet Baysal University. His research interests are audio signal processing, analog and digital filters design.

Arif Nacaroglu was born in Istanbul, Turkey in 1958. He received his B.S., M.S., and Ph.D. degrees in Electrical and Electronics Engineering from METU, Turkey in 1981, 1983, and 1990, respectively. Since1999, he has been a Professor in Electrical and Electronics Engineering in University of Gaziantep. His main research area include switched capacitor networks, time varying systems, analog and digital filters design.
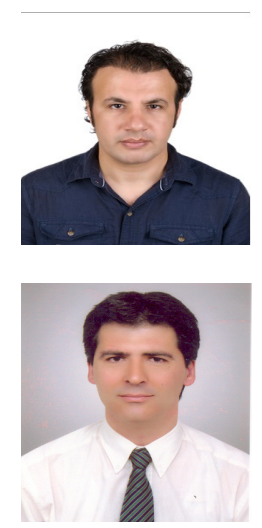